\title{
Modeling Fidelity for Oxygen Nonequilibrium Thermochemistry in Reflected Shock Tube Flows
}

\author{
Kevin Neitzel*, Daniil Andrienko ${ }^{\dagger}$, and Iain D Boyd ${ }^{\ddagger}$ \\ University of Michigan, Ann Arbor, Michigan, 48109, USA
}

\begin{abstract}
The results for a set of vibrational nonequilibrium models with a range of fidelity are compared to experimental data for several post-normal shock test cases. The present work focuses solely on oxygen flows with an emphasis on the modeling of $\mathrm{O}_{2}-\mathrm{O}$. The twotemperature (2T) model is the widely used approach for hypersonic analysis and is presented as the computationally efficient, lower fidelity modeling approach in this work. In contrast, the full state-to-state (STS), master equation approach is presented as the higher fidelity modeling approach. Both approaches have several available methods for obtaining rate data that are investigated. Specifically, the $2 \mathrm{~T}$ model is driven by the rates from Millikan-White (MW) as well as recently available rates that are derived from a detailed quasi-classical trajectory (QCT) analysis for the $\mathrm{O}_{2}-\mathrm{O}$ system. The STS model uses transition rates from the forced harmonic oscillator (FHO) model and dissociation rates from previous work for $\mathrm{O}_{2}-\mathrm{O}$ system. The $\mathrm{O}_{2}-\mathrm{O}$ system uses recently available QCT results for STS transitions and dissociation. The test case results show that capturing non-Boltzmann behavior in the vibrational population distribution is critical to accurate nonequilbirium modeling of hypersonic flows containing oxygen.
\end{abstract}

\section{Nomenclature}

\author{
$A, B$ Millikan-White coefficients \\ $E_{v} \quad$ Vibrational eenergy $[\mathrm{J}]$ \\ $E_{v}^{*} \quad$ Equilibrium vibrational energy $[\mathrm{J}]$ \\ $K \quad$ Reaction rate $\left[\mathrm{cm}^{3} / \mathrm{sec}\right]$ \\ $\mu \quad$ Reduced mass $[\mathrm{kg}]$ \\ $P \quad$ Pressure $[\mathrm{atm}]$ \\ $\rho \quad$ Density $\left[\mathrm{kg} / \mathrm{m}^{3}\right]$ \\ $T_{t} \quad$ Translational temperature $[\mathrm{K}]$ \\ $T_{v} \quad$ Vibrational temperature $[\mathrm{K}]$ \\ $\tau_{v} \quad$ Vibrational relaxation time [sec] \\ $v \quad$ Vibrational quantum state
}

\section{Introduction}

Computer simulation is the primary analysis tool for hypersonic vehicles. The accuracy of the physics modeling dictates the design margin that is required for the vehicle systems, such as the thermal protection system (TPS). Many of the hypersonic flight conditions and geometry do not allow the flow to reach equilibrium before interacting with the vehicle. Modeling this nonequilibrium thermochemistry is one of the main sources of simulation uncertainty. Recent advances in computational power has made it possible to investigate the use of high fidelity modeling methods for hypersonic vehicle analysis.

*PhD Candidate, Department Aerospace Engineering, 1320 Beal Avenue, and Member of AIAA.

${ }^{\dagger}$ Post-doctoral Research Fellow, Department Aerospace Engineering, 1320 Beal Avenue, and Member of AIAA.

$\ddagger$ Professor, Department of Aerospace Engineering, 1320 Beal Avenue, and AIAA Fellow. 
The two-temperature model is the widely used approach for hypersonic vehicle analysis. ${ }^{1}$ For vibrational relaxation, the approach relies on a relaxation time equation that is a function of temperature to evolve the Landau-Teller equation for vibrational energy. The Millikan-White (MW) vibrational relaxation parameter formula is generally used, and correlates well with experimental data for many interactions. ${ }^{2}$ However, predicted vibrational relaxation of the $\mathrm{O}_{2}$-O system does not correlate well with experimental data. ${ }^{3,4}$ The $\mathrm{O}_{2}-\mathrm{O}$ system violates many of the assumptions that are present in the underlying Landau-Teller theory that dictates the temperature dependence of the Millikan-White formula. Park and others have adjusted the Millikan-White coefficients for $\mathrm{O}_{2}$-O to correlate better with experimental data. ${ }^{5}$ However, the previous work has assumed the temperature dependence dictated by the Landau-Teller theory. The vibrational relaxation of $\mathrm{O}_{2}-\mathrm{O}$ is studied in this work using recently developed rates from a detailed quasi-classical trajectory (QCT) analysis. ${ }^{6}$ Chemistry is captured by reaction rates that use Arrhenius-type rate coefficients. Additionally, the Arrhenius form uses the geometrically averaged temperature of the translational and vibrational temperature $\left(T_{a}=\sqrt{T_{t} T_{v}}\right)$ in order to capture nonequilibrium, thermochemical coupling.

The state-to-state (STS) model is a higher fidelity approach to describing the vibrational energy mode. The STS model is much more computationally expensive since the population of each vibrational state is accounted for. The populations are evolved directly based on STS rates and dissociation rates. This approach allows for multi-quantum transitions and non-Boltzmann distributions to be captured. ${ }^{7,8}$ There are two widely used methods for deriving the required STS transition rates. They are the forced harmonic oscillator (FHO) model of Adamovich ${ }^{9-11}$ and the (QCT) analysis. ${ }^{6,15}$ The FHO model is a semi-classical analytical method based on assumptions about the collision event and the form of the potential energy surface (PES). The analytical form of the FHO method makes it very attractive due to the low computational expense required to generate transition rates. The QCT method is more general and can be performed on any PES. The QCT method simulates thousands of individual collision events to calculate transition probabilities and rates for STS transitions and dissociation transitions. The QCT method is computationally expensive, but the recent increase in computational power has made it tractable for some systems. As mentioned, the results of a QCT analysis for $\mathrm{O}_{2}-\mathrm{O}$ are investigated. In particular, the QCT transition rates will be reduced to a vibrational relaxation time, as well as used in a full STS analysis. The $\mathrm{O}_{2}-\mathrm{O}_{2}$ system has not been analyzed using the QCT method to date. The STS rates for $\mathrm{O}_{2}-\mathrm{O}_{2}$ utilize the FHO model.

In summary, the presented work will focus on two nonequilibrium modeling approaches (standard twotemperature model and the state-resolved method). First, the details of the modeling approaches are described. Next, a set of test cases with experimental data is used to compare the different modeling techniques. Finally, the test cases are used to determine requirements of fidelity in nonequilibrium modeling.

\section{Thermochemical Nonequilibrium Model}

In the present work, the post normal shock calculations use the jump conditions derived from the RankineHugoniot relations. The derivation assumes that the electronic and vibrational modes are frozen across the shock wave. The flow downstream of the shock wave is calculated by solving the one-dimensional compressible flow equations plus the equation(s) associated with the vibrational energy mode. The compressible flow equations are shown in Eq. (1).

$$
\frac{\partial}{\partial x}\left(\begin{array}{c}
\rho_{s} u \\
\rho u^{2}+p \\
\rho u\left(h+\frac{1}{2} u^{2}\right)
\end{array}\right)=\left(\begin{array}{c}
m_{s} \omega_{s} \\
0 \\
0
\end{array}\right)
$$

\section{A. Two-Temperature Model}

In the two-temperature (2T) model, nonequilibrium in the energy modes is captured by separating the translation energy, $E_{t}\left(T_{t}\right)$, and the vibrational energy, $E_{v}\left(T_{v}\right)$. The other energy modes are modeled, however they are assumed to be in equilibrium with either the translational or vibrational mode. The present work assumes that the rotational energy mode is in equilibrium with the translational mode and the electronic energy mode is in equilibrium with the vibrational mode. It should also be noted that the present work accounts for only the ground electronic state. Oxygen is known to have accessible, low-lying electronic excited states, however that topic will be the focus of future investigations.

The vibrational energy evolution is governed by the Landau-Teller equation, Eq. (2). 


$$
\frac{d E_{v}}{d t}=\frac{E_{v}^{*}-E_{v}}{\tau_{v}}
$$

The relaxation time, $\tau_{v}$, in Eq. (2) can be obtained from various methods. The most widely accepted method is the Millikan-White relaxation time shown in Eq. (3). The values for A and B are unique for each interaction, and can be calculated based on the reduced mass and vibrational characteristic temperature, $\mathrm{A}=0.00116 \mu^{\frac{1}{2}} \theta^{\frac{4}{3}}$ and $\mathrm{B}=0.015 \mu^{\frac{1}{4}}$.

$$
p \tau_{v}=\exp \left(A\left(T^{-\frac{1}{3}}-B\right)-18.42\right) \quad[a t m-s e c]
$$

The Millikan-White relaxation time is modified with the collision-limited correction term shown in Eq. (4) and Eq. (5). It is also referred to as the high temperature correction (HTC). The correction terms include the number density, $\mathrm{n}$, and the mean particle velocity, c. In this work, $\sigma_{v}^{*}$ is set to $3.0 \times 10^{-21} \mathrm{~m}^{2}$ as proposed by Park.

$$
\begin{gathered}
\tau_{\text {Park }}=\frac{1}{n \sigma_{v} c} \quad[\mathrm{sec}] \\
\sigma_{v}=\sigma_{v}^{*}\left(\frac{50,000}{T}\right)^{2} \quad\left[\mathrm{~m}^{2}\right]
\end{gathered}
$$

The Millikan-White relaxation time equation is derived from Landau-Teller theory. The Landau-Teller theory derives a particular temperature dependence for the relaxation time equation. The Millikan-White relaxation time retains the dominant temperature dependence of the Landau-Teller theory, and is known to describe many species interactions accurately, including the $\mathrm{O}_{2}-\mathrm{O}_{2}$ system. The present work adopts the Millikan-White relaxation time for the $\mathrm{O}_{2}-\mathrm{O}_{2}$ system. ${ }^{5,13}$ However, the assumptions do not apply well to a system containing a molecule and an open shell atom, like the $\mathrm{O}_{2}-\mathrm{O}$ system. ${ }^{3,12,14}$ These types of systems deviate due to the effect of additional mechanisms such as non-adiabatic transitions and and the possibility of atom exchange. The experimental data for the $\mathrm{O}_{2}-\mathrm{O}$ system has been shown to not follow the Millikan-White suggested behavior. Park ${ }^{5}$ proposed new Millikan-White coefficients for $\mathrm{O}_{2}$-O, however, the temperature dependence was still assumed to be consistent with Landau-Teller theory. $\mathrm{O}_{2}-\mathrm{O}_{2}$ has Millikan White coefficients of $\mathrm{A}=135.91$ and $\mathrm{B}=0.030$. The widely accepted Millikan White coefficients for $\mathrm{O}_{2}-\mathrm{O}$ are $\mathrm{A}=47.7$ and $\mathrm{B}=0.0271$.

One of the main areas of focus for this work is to apply the newly available QCT calculated transition rates for $\mathrm{O}_{2}-\mathrm{O}$ from Andrienko and Boyd. ${ }^{6}$ Two sets of state-to-state transition rates are constructed. First, a simplified Hulbert-Hirshfelder (H-H) PES is applied in a pairwise fashion. The PES supports a maximum of 36 vibrational states and 223 rotational levels for electronic ground-state $\mathrm{O}_{2}$. This simplified $\mathrm{H}-\mathrm{H}$ PES is computationally less expensive than a more accurate PES, and has been shown to successfully capture the rovibrational relaxation of $\mathrm{O}_{2}$-Ar. Second, an accurate many-body PES is adopted. The Varandas and Pais $\mathrm{PES}^{6}$ generates 47 vibrational states and a maximum of 236 rotational levels for electronic ground-state molecular oxygen. Equation (6) presents the curve fit form of the vibrational relaxation parameter for the QCT results. Further details on the QCT analysis can be found in Ref. 6.

$$
P \tau_{v i b}=\left(a x^{3}+b x^{2}+c x+d\right) \times 10^{-8} \quad[a t m-s e c]
$$

Table 6 summarizes the curve fit coefficients for each QCT PES.

\begin{tabular}{ccccc}
\hline & $\mathrm{a}$ & $\mathrm{b}$ & $\mathrm{c}$ & $\mathrm{d}$ \\
\hline HH PES & $-4.407 \times 10^{-6}$ & -0.005662 & 0.5433 & 0.08702 \\
Varandas PES & $2.304 \times 10^{-3}$ & -0.07254 & 1.245 & 1.70 \\
\hline
\end{tabular}

Table 1. Vibrational relaxation curve fit coefficients obtained from QCT analyses

Figure 1 compares the vibrational relaxation parameter from different methods for $\mathrm{O}_{2}-\mathrm{O}\left(\mathrm{O}_{2}-\mathrm{O}_{2}\right.$ is also shown for reference). There is much insight that can be gained from this plot. First, the unadjusted MW relaxation parameter for $\mathrm{O}_{2}$-O does not match up well with the experimental data of Breen. ${ }^{16}$ This is what lead Park to adjust the MW coefficients to match the experimental data. The experimental data for $\mathrm{O}_{2}-\mathrm{O}$ is only available at relatively low temperatures (less than 4000K), which makes it difficult to infer the correct 
temperature dependence. Park and others have assumed the same form as the Landau-Teller temperature dependence. No temperature dependence is assumed in the QCT method. Secondly, the QCT results deviate from the Landau-Teller temperature dependence. This is plausible, if not expected, given that the $\mathrm{O}_{2}-\mathrm{O}$ system does not adhere to the assumptions of Landau-Teller theory.

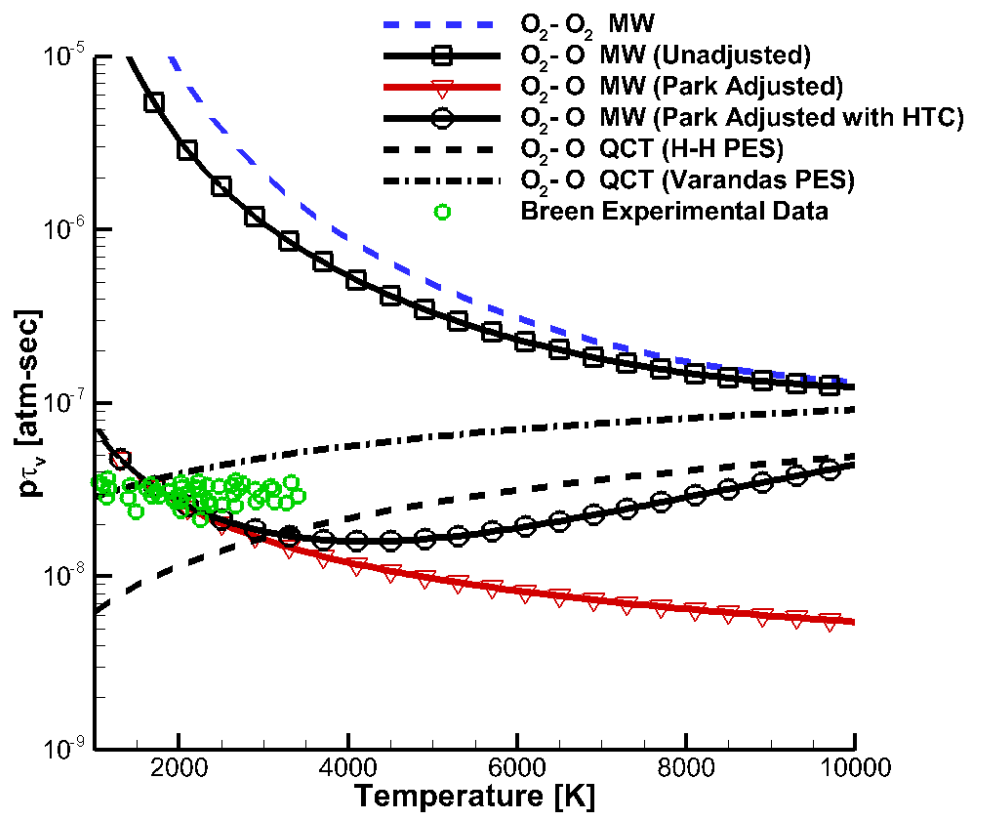

Figure 1. Vibrational relaxation parameter obtained from various models

In the $2 \mathrm{~T}$ model, the dissociation process is treated by Arrhenius-type rate coefficients with the geometrically averaged temperature of the translational and vibrational temperature $\left(T_{a}=\sqrt{T_{t} T_{v}}\right)$. The Arrhenius form is shown in Eq. 7. Additionally, the Arrhenius coefficients associated with various models are summarized in Table 2. When a molecule dissociates, its vibrational energy is lost, and this effect must be accounted for in the model. Since the dissociating molecule could be at a low vibrational state or a high vibrational state, an assumption must be made as to an average energy loss due to dissociation. The present work assumes this energy loss due to dissociation to be 0.45 of the dissociation energy.

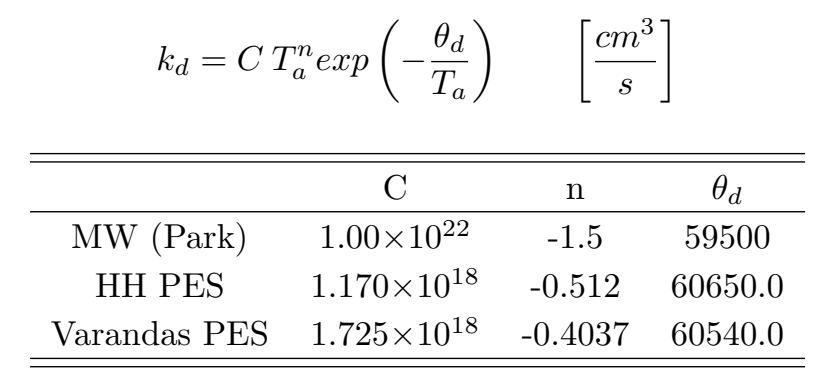

Table 2. Arrhenius parameters of dissociation reaction, pre-exponential factor is in $\mathrm{cm}^{3} / \mathrm{s} / \mathrm{mole}$

\section{B. State-To-State Model}

A detailed QCT analysis was performed by Andrienko and Boyd ${ }^{6}$ for the $\mathrm{O}_{2}-\mathrm{O}$ system. The state-to-state transition rates and dissociation rates are reduced to a relaxation time and dissociation rate through a series of master equation analyses. The final results are provided in a curve fit form as shown in Eq. 8 and Eq. 9. The transition rates for the $\mathrm{O}_{2}-\mathrm{O}_{2}$ system are taken from FHO analysis. ${ }^{9-11,13}$ These rates are 
used in a post-normal shock calculation using the master equation approach outlined in Ref. 8. It can be noted that a STS model explicitly represents the populations of each vibrational state. The evolution of the population distribution is driven by the STS rates and dissociation rates. There is no assumption made for the population distribution or the coupling between the vibrational relaxation and dissociation.

$$
\begin{gathered}
K_{v \rightarrow v^{\prime}}=1 \times 10^{-12} \exp \left[A+\frac{B}{\log (x)}+C \log (x)\right] \quad x=\frac{T}{1000} \quad\left[\frac{\mathrm{cm}^{3}}{\mathrm{~s}}\right] \\
\log \left(K_{v \rightarrow \text { diss }}\right)=A+B \log (T)-\frac{C}{T} \quad\left[\frac{\mathrm{cm}^{3}}{\mathrm{~s}}\right]
\end{gathered}
$$

\section{Post Normal Shock Flow Results}

\section{A. Two-Temperature Results}

The post normal shock flow calculations are carried out for an existing set of shock tube experiments conducted by Shatalov et al. ${ }^{17}$ The flow conditions for the test cases are summarized in Table 3. All flow conditions have a pure $\mathrm{O}_{2}$ freestream composition.

\begin{tabular}{ccccc}
\hline \hline Test Case & Shock Velocity $\left[\frac{\mathrm{km}}{\mathrm{s}}\right]$ & $\mathrm{P}_{1}[$ Torr $]$ & $\mathrm{T}_{1}$ & $\mathrm{~T}_{\text {Post }}[\mathrm{K}]$ \\
\hline $\mathrm{C} 1$ & 3.07 & 2.0 & 295 & 5,300 \\
$\mathrm{C} 2$ & 3.95 & 1.0 & 295 & 8,620 \\
$\mathrm{C} 3$ & 4.44 & 0.8 & 295 & 10,820 \\
\hline \hline
\end{tabular}

Table 3. Summary of flow conditions investigated ${ }^{17}$

Figure 2 shows the temperature profiles of the various 2T models for Case C1. All of the models perform nearly identically. $\mathrm{C} 1$ is a mild condition with a relatively low post-shock temperature. The mild temperature leads to a very small amount of atomic oxygen being present during the vibrational relaxation. Figure 3 shows the composition evolution. Note the difference in time scale for the composition plot. Since there is little to no atomic oxygen present during the vibrational relaxation, the $\mathrm{O}_{2}-\mathrm{O}_{2}$ behavior dominates. All of the models are utilizing the same MW coefficients for $\mathrm{O}_{2}-\mathrm{O}_{2}$, so it is not a surprise that they all have almost identical behavior. Only slight differences are observed after $0.5 \mu$ sec due to the start of dissociation, and thus the presence of atomic oxygen. It is also observed that the results from all models fall within the error bars of the experimental data.

Figure 4 shows the temperature profiles and Fig. 5 shows the composition evolution for Case C2. For this condition, dissociation occurs during the vibrational relaxation process. This introduces atomic oxygen that influences the vibrational relaxation process, and slight differences are seen in the prediction of the different models. Specifically, after $0.2 \mu$ sec the behavior differs significantly due to the different dissociation models. The results generally agree with experimental data until $0.25 \mu \mathrm{sec}$. After this point, the models all under predict the vibrational temperature.

The temperature profiles for the model results for Case C3 are shown in Fig. 6. Once again the results from all the models have very similar behavior. They also agree well with the experimental data. Case C3 has the highest temperature of the test cases. In this high temperature scenario, the vibrational relaxation occurs very quickly, more rapidly than the dissociation process (see Fig. 7). This leads to the end result being similar to Case $\mathrm{C} 1$ in which the vibrational relaxation is completed before any atomic oxygen is present. This leads to $\mathrm{O}_{2}-\mathrm{O}_{2}$ dominating the vibrational relaxation behavior. The differences in the behavior is primarily from the dissociation modeling.

\section{B. State-to-State Results}

The results in this section focus on comparing the current state of the art method (2T with Millikan-White) with the highest fidelity model in this study (STS with QCT Varandas rates).

Figure 8 presents the evolution of temperature for the two modeling approaches for Case C1. There is a fundamental difference in the vibrational temperature profile. The STS QCT Varandas vibrational temperature results begin rising more slowly than the Millikan-White results. However, the STS QCT 


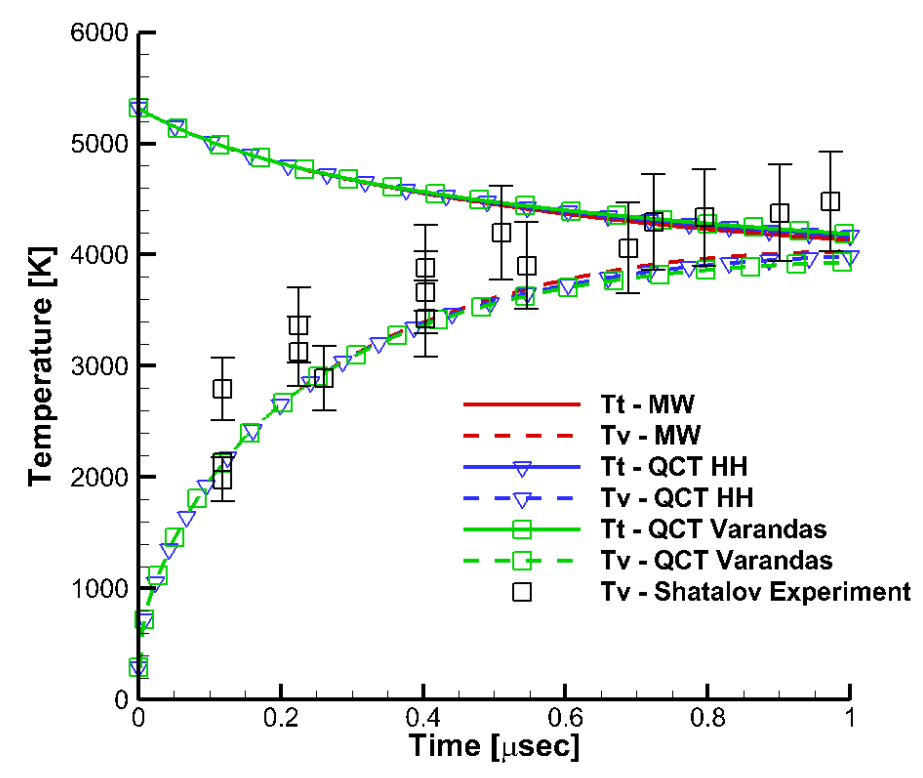

Figure 2. Temperature profiles obtained with different relaxation models for C1: $T_{1}=295 K, P_{1}=2.0 T o r r, ~ V=3.07 \mathrm{~km} / \mathrm{s}$, Pure $\mathbf{O}_{2}$ freestream

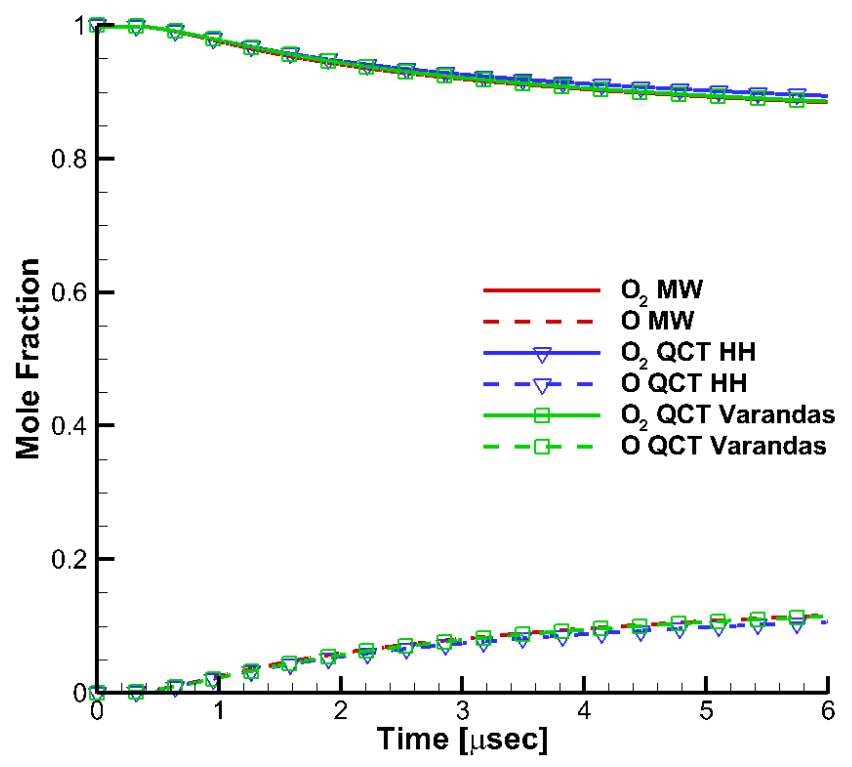

Figure 3. Composition profile for C1: $T_{1}=295 K, P_{1}=2.0 T o r r, V=$ $3.07 \mathrm{~km} / \mathrm{s}$, Pure $\mathbf{O}_{2}$ freestream

Varandas results have a more linear profile early, and crosses over the MW profile around $0.4 \mu$ sec. Both models follow the low end of the experimental data error band. Prior to $0.4 \mu \mathrm{sec}$, the MW results follow the experimental data more closely. Conversely, the STS QCT Varandas results follow the experimental data better after $0.4 \mu$ sec. Figure 9 shows the difference in composition and may account for the differences 


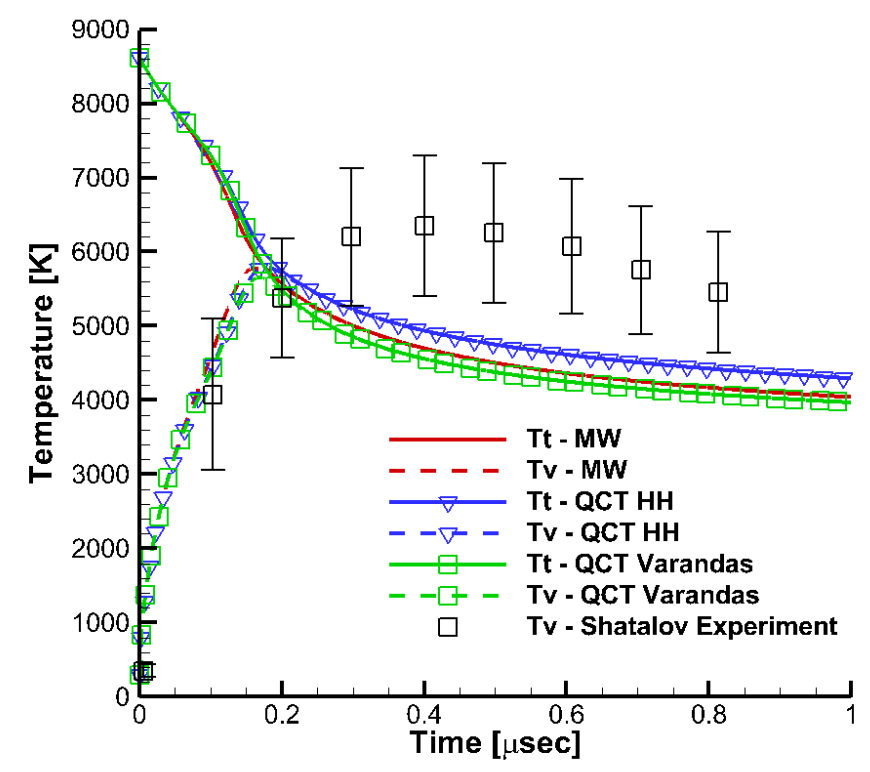

Figure 4. Temperature profiles obtained with different relaxation models for C2: $T_{1}=295 \mathrm{~K}, P_{1}=1.0 T o r r, V=3.95 \mathrm{~km} / \mathrm{s}$, Pure $\mathbf{O}_{2}$ freestream

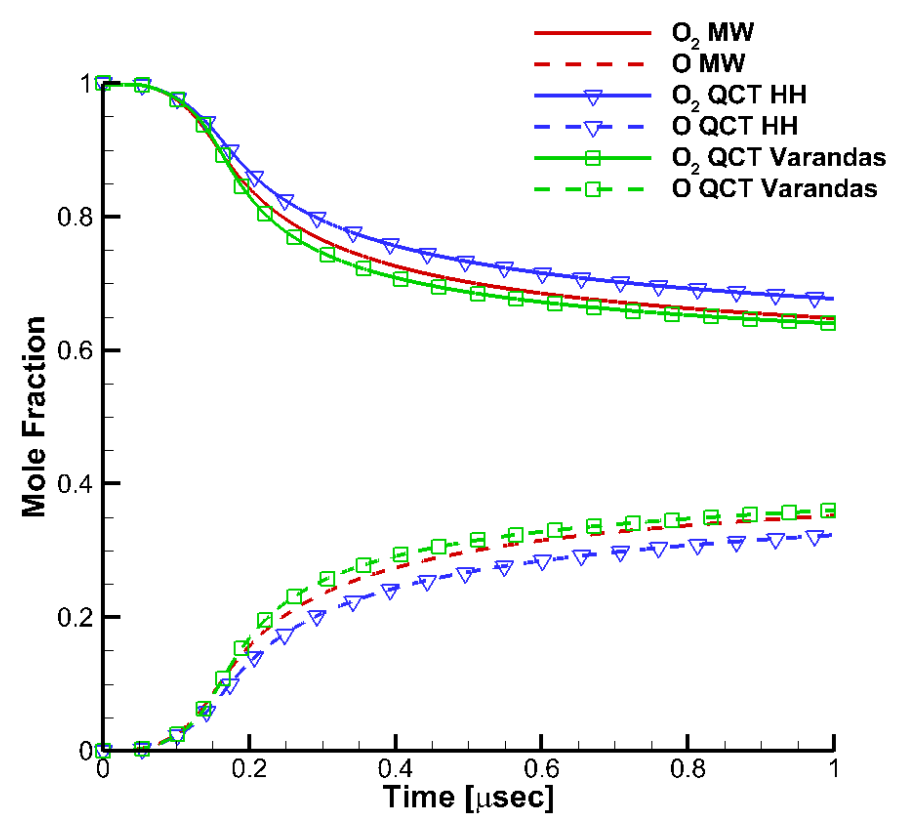

Figure 5. Composition profile for C2: $T_{1}=295 \mathrm{~K}, \mathrm{P}_{1}=1.0 \mathrm{Torr}, \mathrm{V}=$ $3.95 \mathrm{~km} / \mathrm{s}$, Pure $\mathbf{O}_{2}$ freestream

observed in the vibrational temperature profile. While there are differences in the fundamental profile shapes of the two models, neither model shows a distinct advantage in replicating the experimental data for vibrational temperature. Figure 10 presents the evolution of vibrational state population distribution for the STS model. The Boltzmann distributions plotted represent a temperature equivalent distribution 


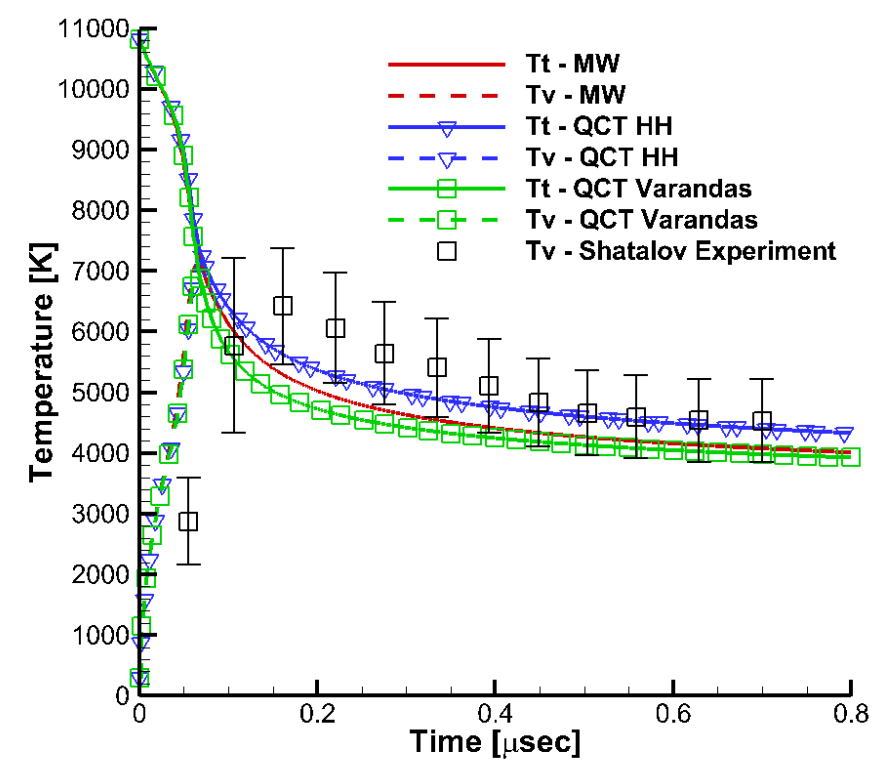

Figure 6. Temperature profiles obtained with different relaxation models for C3: $T_{1}=295 \mathrm{~K}, P_{1}=0.8 \mathrm{Torr}, \mathrm{V}=4.44 \mathrm{~km} / \mathrm{s}$, Pure $\mathbf{O}_{2}$ freestream

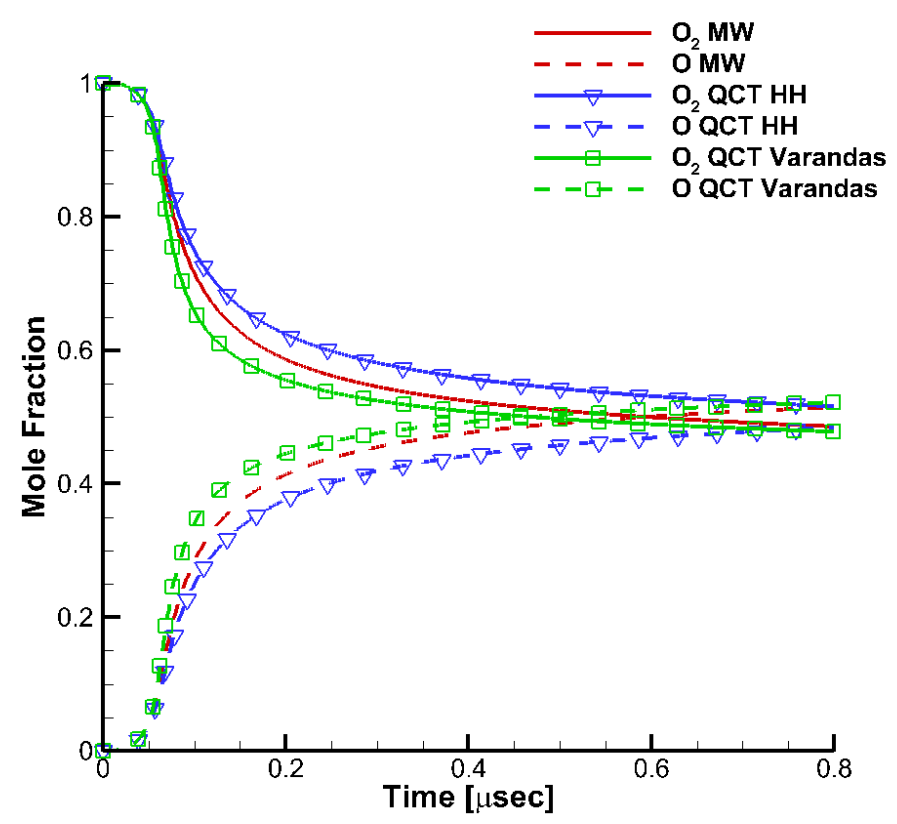

Figure 7. Composition profile for C3: $T_{1}=295 K, P_{1}=0.8 T o r r, V=$ $4.44 \mathrm{~km} / \mathrm{s}$, Pure $\mathbf{O}_{2}$ freestream

for that given time. The first time location $(\mathrm{t}=0.00 \mu \mathrm{sec})$ represents the vibrational population distribution just before the shock passage. The flow is in equilibrium and the distribution does not deviate from the Boltzmann distribution. However, once the shock passes, the distribution is non-Boltzmann throughout the vibrational relaxation and dissociation processes. Specifically, the distribution is overpopulated (relative to 
the Boltzmann distribution) for the mid and high level states early in the evolution, while the vibrational relaxation is the dominant process. The overpopulation begins to be mitigated as the dissociation process becomes more significant. When comparing the $0.04 \mu \mathrm{sec}$ and $0.36 \mu \mathrm{sec}$ distributions, the effect of dissociation has begun to diminish the overpopulation at the high level states. This effect continues into the 0.75 $\mu$ sec distribution. At this time, the distribution is primarily Boltzmann except for the high energy tail that is underpopulated due to the dissociation process. This distribution profile remains until the species populations settle out and the system reaches equilibrium. The end equilibrium vibrational population distribution is omitted from the figure for clarity. Similar qualitative evolution behavior is observed in $\mathrm{C} 2$ and C3 for the vibrational population distribution.

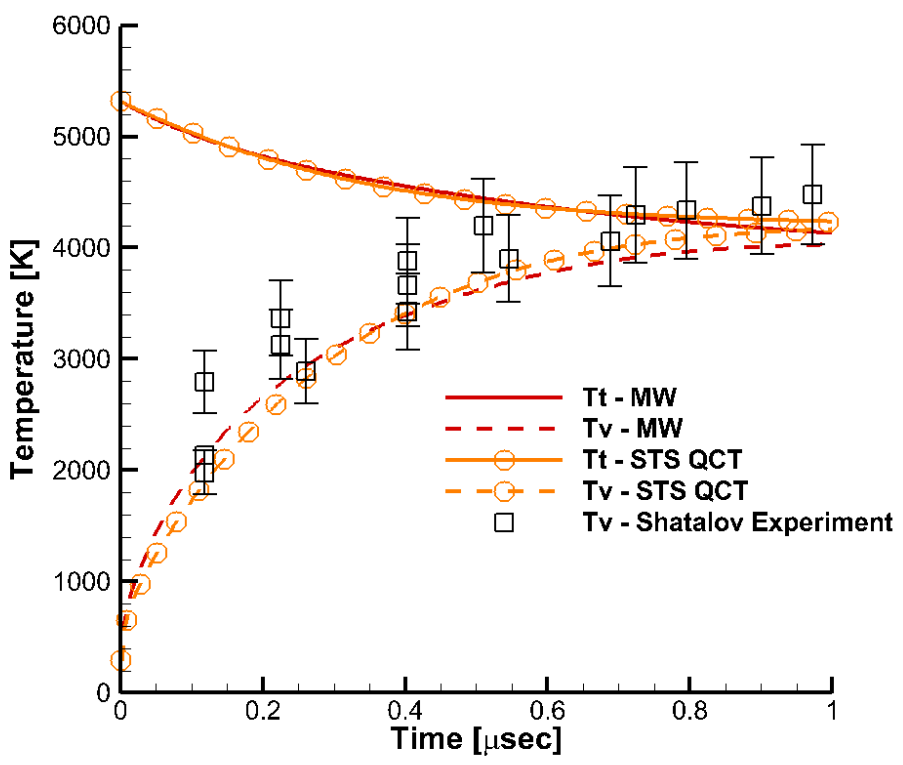

Figure 8. Temperature profiles obtained with different relaxation models for $\mathrm{C} 1$

Figure 11 and 12 present the evolution of temperature and composition for the two modeling approaches for Case C2. The difference in the behavior between the two models is significant. Case C2 is interesting in that the dissociation process and the vibrational relaxation are occurring simultaneously. It appears that the ability of the STS model to describe non-Boltzmann behavior and multi-quantum transitions is critical to properly modeling flows with this vibrational relaxation and dissociation process interaction. Figure 10 presents the vibrational population distribution. The behavior is similar to that described for Case C1.

Figure 14 and 15 present the evolution of temperature and composition for the two modeling approaches for Case C3. The difference in the behavior between the two models is significant once again. Case C3 also has the dissociation process and the vibrational relaxation are occurring simultaneously. The results are similar to Case C2. The STS model can describe the behavior more accurately than the 2T model. Figure 16 presents the vibrational population distribution. The behavior is similar to that described for Case C1.

It should be noted that the non-Boltzmann behavior observed in the vibrational population distributions is linked to the profile shape differences observed in vibrational temperature evolution. The $2 \mathrm{~T}$ method assumes a Boltzmann distribution throughout the process as well as single quantum transitions. Theses assumptions lead to a smoother (more constant curvature) profile. The STS model allows for non-Boltzmann distributions and multi-quantum transitions that lead to different relaxation and dissociation behavior, causing additional inflection points in the vibrational temperature profile. Similar behavior has been observed for nitrogen. ${ }^{18}$ 


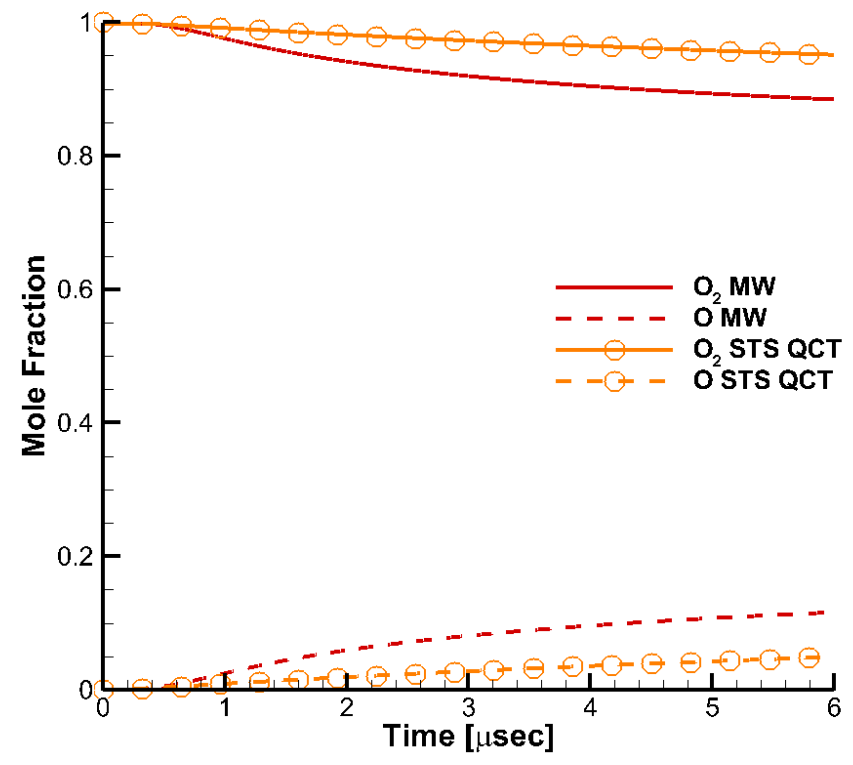

Figure 9. Composition profiles obtained with different relaxation models for C1 (Note the timescale difference)

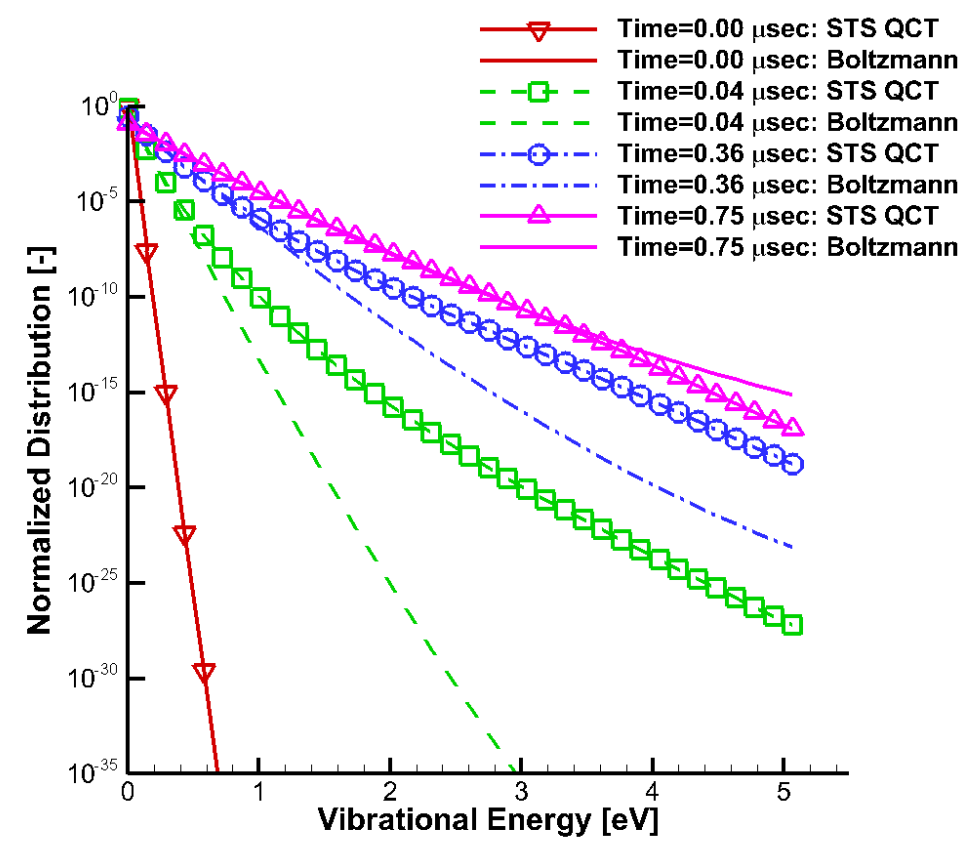

Figure 10. Vibrational population distribution evolution for C1

\section{Conclusion}

Vibrational nonequilibrium and dissociation models of varying fidelity are presented in this work. First, the widely used two-temperature model is presented with various potential vibrational relaxation rate and dissociation rate expressions. The results show only small differences between the different vibrational relaxation rate methods for $\mathrm{O}_{2}$-O. The results suggest that the differences in behavior is due to the dissociation 


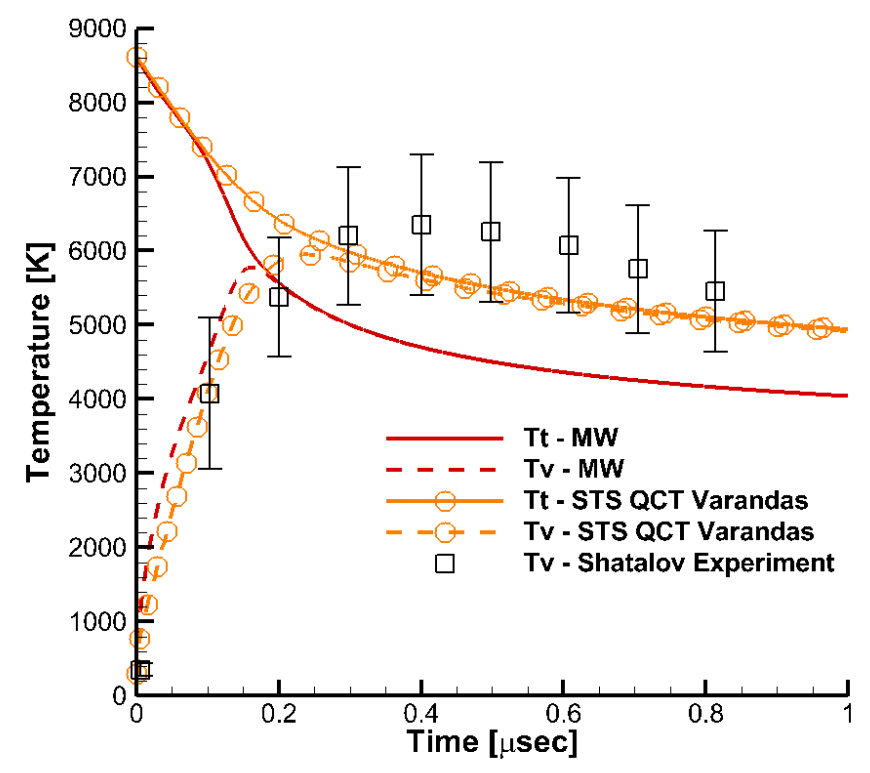

Figure 11. Temperature profiles obtained with different relaxation models for C2

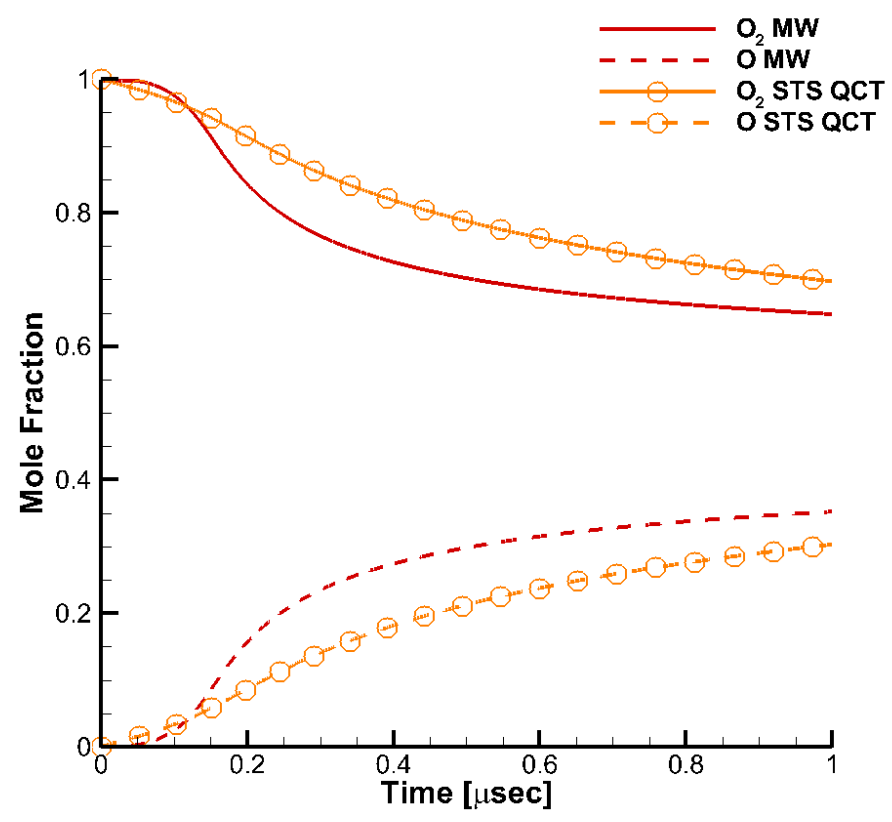

Figure 12. Composition profiles obtained with different relaxation models for C2

rates for the various models. Two vibrational relaxation rate functions are presented that were obtained from QCT analysis of two different PES. The test case results show a slight difference between the two different PES, but not a fundamental difference in behavior. Additionally, detailed state-to-state calculations were performed using the transition rates from the FHO model and QCT analysis for $\mathrm{O}_{2}-\mathrm{O}_{2}$ and $\mathrm{O}_{2}-\mathrm{O}$, respectively. The investigation showed that there is strong non-Boltzmann behavior in the vibrational population 


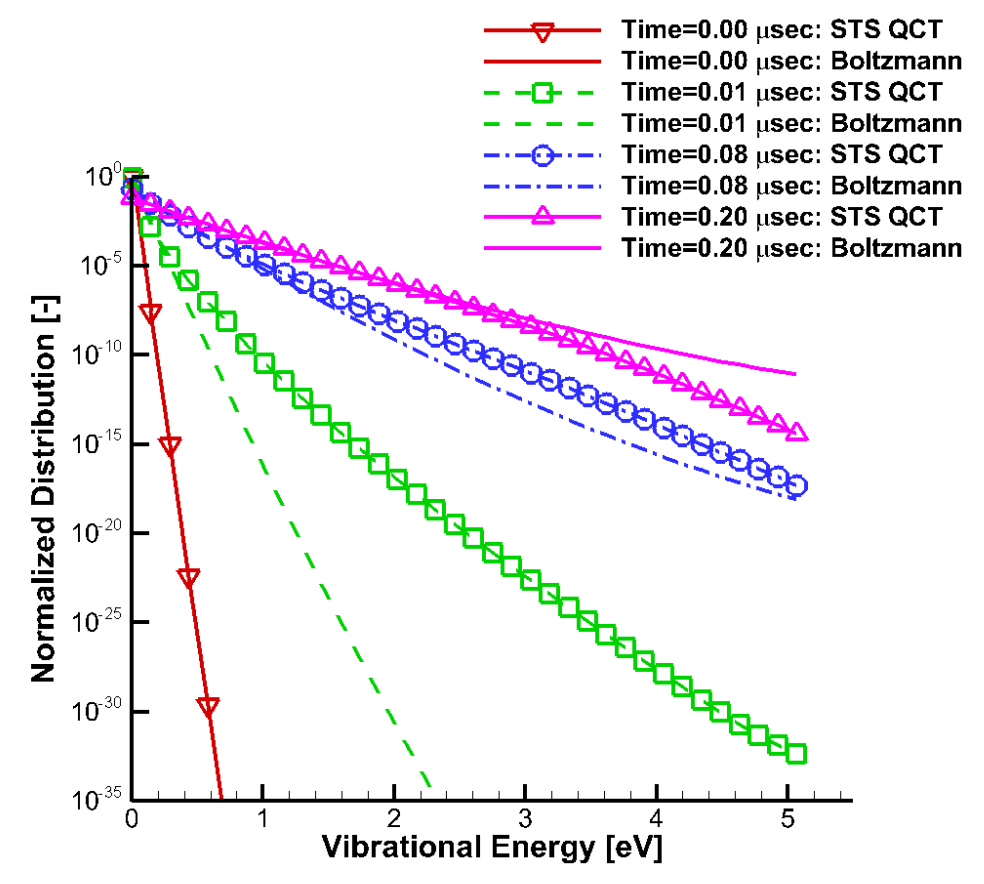

Figure 13. Vibrational population distribution evolution for C2

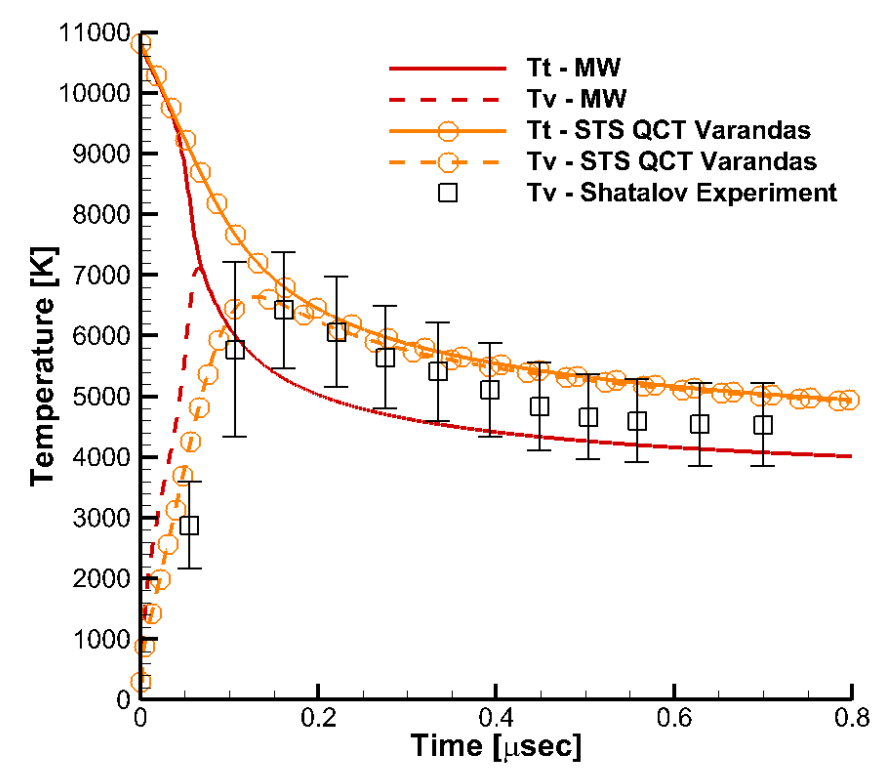

Figure 14. Temperature profiles obtained with different relaxation models for C3

distributions for the cases studied, specifically C2 and C3. The results show that there need to capture nonBoltzmann behavior and multiquantum transitions for flow conditions that have both vibrational relaxation and dissociation occurring simultaneously. Additionally, the importance of accurate STS transition rates for $\mathrm{O}_{2}-\mathrm{O}_{2}$ is highlighted. 


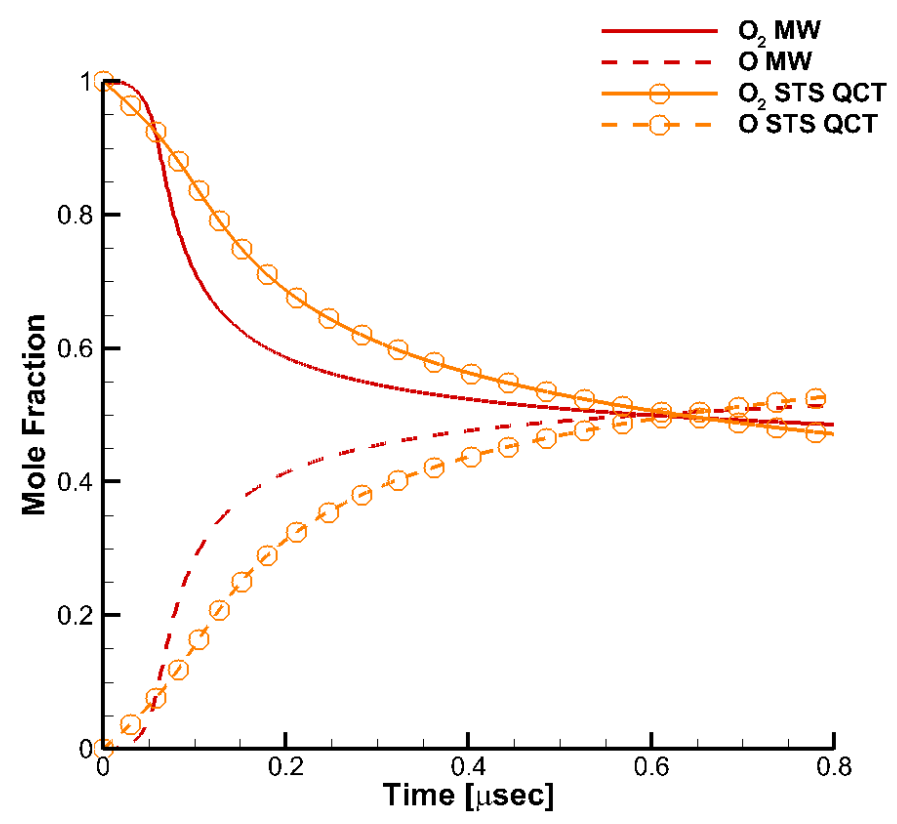

Figure 15. Composition profiles obtained with different relaxation models for C3

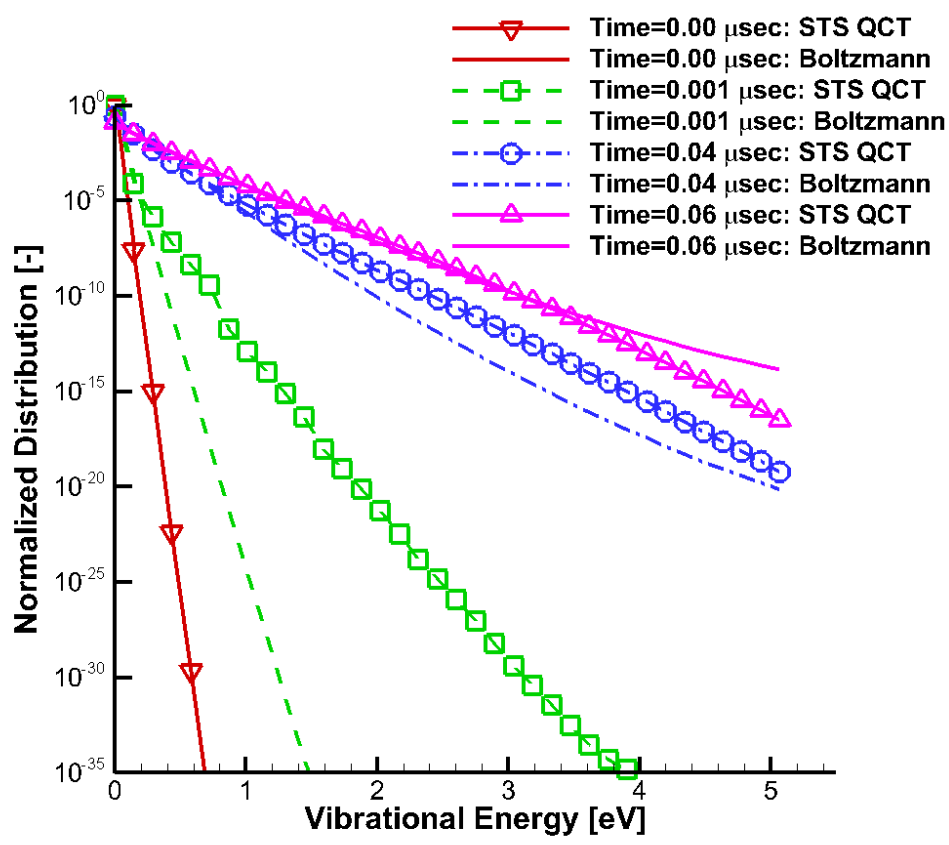

Figure 16. Vibrational population distribution evolution for C3

\section{Acknowledgments}

The authors gratefully acknowledge funding for this work through Air Force Office of Scientific Research Grant FA9550-12-1-0483. KN would like to thank Dr. Jae Gang Kim for the program code that would evolve into the flow solver used in this work. 


\section{References}

${ }^{1}$ C. Park, "Nonequilibrium Hypersonic Aerothermodynamics," Wiley, New York, 1990, pp. 119-144.

${ }^{2}$ R.C. Millikan and D.R. White, "Systematics of vibrational relaxation," The Journal of Chemical Physics, Vol. 39, No. 12, p. $3209,1963$.

${ }^{3}$ F. Thivet, M.Y. Perrin, S. Candel, "A unified nonequilibrium model for hypersonics," Physics of Fluids, Vol. 3, p. 2799, 1991.

${ }^{4}$ M. Holden, T. Wadhams, M. MacLean, "A Review of Experimental Studies with the Double Cone and Hollow Cylinder/Flare Configurations in the LENS Hypervelocity Tunnels and Comparisons with Navier-Stokes and DSMC Computations," AIAA 2010-1281, 48th AIAA Aerospace Sciences Meeting, Orlando, Florida, January 2010.

${ }^{5}$ C. Park, "Review of Chemical-Kinetic Problems of Future NASA Missions, I: Earth Entries," Journal of Thermophysics and Heat Transfer. Vol. 7, No. 3, 1993, pp. 385-398.

${ }^{6}$ D. Andrienko, I.D. Boyd, "Vibrational Relaxation and Dissociation of Oxygen in Molecule-Atom Collisions," 46th AIAA Thermophysics Conference, AIAA Paper 2015-XXXX, June 2015.

${ }^{7}$ K. Neitzel, J.G. Kim, I.D. Boyd, "Nonequilibrium Modeling of Oxygen in Reflected Shock Tube Flows," 45th AIAA Thermophysics Conference, AIAA Paper 2014-2961, June 2014.

${ }^{8}$ J.G. Kim and I.D. Boyd, "State-resolved master equation analysis of thermochemical nonequilibrium of nitrogen," Chemical Physics, Vol. 415, p. 237-246, 2013.

${ }^{9}$ I.V. Adamovich and J.W. Rich, Threedimensional nonperturbative analytical model of vibrational energy transfer in atom-molecule collisions, Journal of Chemical Physics, Vol. 109, No. 18, 1998, pp. 7711-7724.

${ }^{10}$ I.V. Adamovich, S.O. Macheret, J.W. Rich, and C.E. Treanor, Vibrational Relaxation and Dissociation Behind Shock Waves Part 2: Master Equation Modeling, Journal of Thermophysics and Heat Transfer, Vol. 33, No. 6, 1995, pp. $1070-1075$.

${ }^{11}$ I.V. Adamovich, Three-dimensional Analytic Model of Vibrational Energy Transfer in Molecule-Molecule Collisions, AIAA Journal, Vol. 39, No. 10, 2001, pp. 1916-1925.

${ }^{12}$ F. Esposito, I. Armenise, G. Capitta, M. Capitelli, "O-O $\mathrm{O}_{2}$ state to state vibrational relaxation and dissociation rates based on quasiclassical calculations," Chemical Physics. Vol. 351, 2008, pp. 91-98.

${ }^{13}$ M. Lino da Silva, J. Loureiro, V. Guerra, A multiquantum dataset for vibrational excitation and dissociation in hightemperature $\mathrm{O}_{2}-\mathrm{O}_{2}$ collisions, Chemical Physics Letters, Vol. 531, 2012, pp. 28-33.

${ }^{14}$ F. Esposito and M. Capitelli, "The relaxation of vibrationally excited $\mathrm{O}_{2}$ molecules by atomic oxygen," Chemical Physics Letters, Vol. 443, p. 222-226, 2007.

${ }^{15}$ F. Esposito, I. Armenise, G. Capitta, and M. Capitelli, "O $+\mathrm{O}_{2}$ state-to-state vibrational relaxation and dissociation rates based on quasiclassical calculations," Chemical Physics, Vol. 351, pp. 91-98, July 2008.

${ }^{16} \mathrm{~J}$. Breen, R. Quy, G. Glass, "Vibrational relaxation of $\mathrm{O}_{2}$ in the presence of atomic oxygen," The Journal of Chemical Physics, Vol. 59, p. 556-557, 1973.

${ }^{17}$ L.B. Ibraguimova, A.L. Sergievskaya, V.Yu. Levashov, O.P. Shatalov, Yu.V. Tunik, "Investigation of oxygen dissociation and vibrational relaxation at temperatures 4,000-10,800K," The Journal of Chemical Physics, Vol. 139, p. 034317-034328, 2013.

${ }^{18}$ B. Lopez, M. Lino da Silva, "Non-Boltzmann Analysis of Hypersonic Air Re-Entry Flows," AIAA Paper 2014-1889955, June 2014.

${ }^{19}$ R. Jongma, A. Wodtke, "Fast multiquantum vibrational relaxation of highly vibrationally excited $\mathrm{O}_{2}$," The Journal of Chemical Physics, Vol. 111, p. 10957-10963, 1999. 